\title{
Chromosomes et cancer Le paradigme du lymphome de Burkitt
}

\section{Jean-Claude Kaplan \\ Professeur de biochimie médi- cale à l'UER Cochin-Port- Royal.}

\section{Marie-France Szajnert} Docteur d'Université Paris $V$.

\section{RÉFÉRENCES}

I. Burkitt D. A sarcoma involving the jaw in African children. Br 7 Surg 1958; $46: 218-23$.

2. Epstcin M., Achong B., Barr Y. Virus particles in cultured lymphoblasts from Burkitt's lymphoma. Lancet 1964; i : 702-3

3. Lenoir G. M., Philip T., Sohier R. Burkitttype lymphoma: EBV association and cytogenetic markers in cases from various geographic locations. In: Magrath I. T., O'Connor G. T., Ramot B., éds. Pathogenesis of leukemias and lymphomas: environmental influences, New York: Raven Press, 1984: 283-5.

4. Thé G. (de), Geser A., Day N. E. Epidemiological evidence for causal relationship between Epstein-Barr virus and Burkitt's lymphoma from Ugandan prospective study. Nature 1978; 274: 756-6r.

\section{ADRESSE}

J. C. Kaplan, M. F. Szajnert : Institut dc pathologie moléculaire. Inserm (U I 29), 24, rue du Faubourg Saint-Jacques, 75014 Paris.
$1 \mathrm{y}$ a près de 30 ans, Burkitt publiait sa description princeps d'un lymphosarcome de la mâchoire frappant l'enfant africain avec une remarquable fréquence. $\mathrm{La}$ maladie, d'abord observée en Ouganda, fut ensuite signalée dans d'autres régions de l'Afrique centrale et orientale, ainsi qu'en Nouvelle-Guinée [I]. En I964, Epstein et Barr décrivaient dans des lignées lymphoïdes continues, établies à partir de matériel tumoral, un virus nouveau, appartenant à la catégorie des virus herpétiques, qui devait recevoir leur nom [2]. Le lymphome de Burkitt a donc d'abord été observé dans des régions où sévit le paludisme et où les conditions socio-économiques favorisent une infestation précoce de toute la population par le virus d'Epstein-Barr (EB). En fait, on sait maintenant que le lymphome de Burkitt est aussi observé de façon sporadique en dehors des régions tropicales. En France il représente un tiers des lymphomes malins non hodgkiniens de l'enfant, soit $3 \%$ des cancers de l'enfant [3].

Dans les régions endémiques (Afrique centrale et Nouvelle-Guinée) où le lymphome de Burkitt frappe annuellement 4 enfants sur roo o0o, il est associé au virus EB dans presque tous les cas [4]. Dans les autres régions, où l'incidence est 20 fois plus faible, l'association au virus EB n'est retrouvée que dans $10 \%$ des cas. Dans les populations à standard socio-économique élevé, la primoinf estation par le virus EB est soit latente, soit génératrice d'une maladie bénigne : la mononucléose infectieuse. La découverte d'anomalies chromosomiques constantes et caractéristiques a grandement contribué à unifier le concept de lymphome de Burkitt, qu'il soit endémique ou sporadique.
Au début des années quatre-vingts, les connaissances sur le lymphome de Burkitt se limitaient aux notions suivantes : il s'agit d'une prolifération maligne des lymphocytes B; elle est monoclonale; elle s'accompagne dans $100 \%$ des cas d'une translocation $t(8 ; 14), t(8 ; 22)$ ou $t(2 ; 8)$; elle peut être associée au virus $\mathrm{EB}$, sans que l'on sache le rôle exact de celui-ci. Le lymphome de Burkitt n'a pas manqué de bénéficier des progrès de la biologie liés à l'introduction des méthodes du génie génétique, et il est même devenu un modèle d'étude tout à fait privilégié. Il permet en effet d'étudier les relations entre remaniements chromosomiques et cancers; de jeter un pont entre le domaine de la cytogénétique (niveau de définition minimale sur l'ADN $=5$ millions de bases) et celui de la biologie moléculaire (niveau d'appréhension maximale $=50000$ bases); de rechercher un éventuel effet de position génique non encore démontré dans l'espèce humaine; de chercher une éventuelle intervention d'oncogènes, nouvelle catégorie de gènes impliqués dans le cancer; d'éclaircir le mécanisme de la synthèse des immunoglobulines; d'envisager le problème de la différenciation des lymphocytes B. Un dernier atout, non négligeable, de ce modèle est de bénéficier d'un matériel d'étude abondant constitué par des lignées clonales faciles à cultiver en suspension.

$\mathrm{Au}$ Centre International de Recherche sur le Cancer, à Lyon, il existe un laboratoire de référence pour l'étude du lymphome de Burkitt. Il joue un rôle important en centralisant les données et en établissant systématiquement des lignées à partir du matériel qu'il reçoit.

Les lignées de Burkitt portent 


\section{RÉFÉRENCES}

5. Zech L., Haglund V., Nilsson K., Klein G Characteristic chromosomal abnormalities in biopsies and lymphoid cell lines from patients with Burkitt and non-Burkitt lymphomas. Int $\mathrm{J}$ Cancer 1976; $17: 47-56$

6. Bernheim A., Berger R., Lenoir G. Cytogenetic studies on African Burkitt's lymphoma cell lines: $t(8 ; 14), t(2 ; 8)$ and $t(8 ; 22)$ translocations Cancer Genet Cytogenet 1981; 3:307-1 5 .

7. Bernheim A., Berger R., Lenoir G. Cytogenetic studies on Burkitt's lymphoma cell lines. Cancer Genet Cytogenet 1983; 8:223-9.

8. Ohno S., Babonits M., Wiener F., Spira J., Klein G., Potter M. Non-random chromosome changes involving the Ig gene-carrying chromosomes 12 and 6 in pristane-induced mouse plasmocytomas. Cell 1979; 18 : 1001-7.

9. Lenoir G. M., Preud'homme J. L., Bernheim A., Berger R. Correlations between immunoglobulin light chain expression and variant translocation in Burkitt's lymphoma. Nature 1982; $298: 474-6$.

Io. Erikson J., Finan J., Nowell P. C., Croce C. M. Translocation of immunoglobulin $\mathrm{VH}$ genes in Burkitt lymphoma. Proc Natl Acad Sci USA 1982; 79: 5611-5.

II. de La Chapelle A., Lenoir G., Boué J. et al. Lambda Ig constant region gene are translocated to chromosome 8 in Burkitt lymphoma with (8; 22). Nucleic Acids Res 1983; 11 : $1133-42$.

12. Erikson J., Nishikura K., ar-Rushdi A. et al. Translocation of an immunoglobulin kappa locus to a region 3 ' of an unrearranged $c$-myc oncogene enhancer $c$-myc transcription. Proc Natl Acad Sci $U S A$ 1983; $80: 7581-85$

13. Leder $\mathbf{P}$. The genetics of antibody diversity. Sci Am 1982; 246:72-83.

14. Hayward W. S., Neel B. G., Astrin S. M Activation of cellular oncogenes by promoter insertion in ALV-induced lymphoid leukosis. Nature 1981; 296 : 475-9.

15. Taub R., Kirsch I., Morton C. et al. Translocation of the $c$-myc gene into the immunoglobulin heavy chain locus in human Burkitt lymphoma and murine plasmocytoma cells. Proc Natl Acad Sci US A 1982; 79: 7837-4 I.

16. Erikson J., ar-Rushdi A., Drwinga H. L. Nowell P. C., Croce C. M. Transcriptional activation of the translocated $c$-myc oncogene in Burkit lymphoma. Proc Natl Acad Sci USA-1983; $80: 820-4$.

17. Dalla Favera R., Martinotti S., Gallo R. C. Erikson J., Croce C. M. Translocation and rearrangements of the $c$-myc oncogene locus in human undifferentiated B-cell lymphomas. des remaniements chromosomiques plus ou moins complexes où s'individualisent des anomalies constantes et significatives [5-7]. Dans environ $70 \%$ des cas, il existe une translocation équilibrée entre le chromosome 8 et le chromosome I4, $t(8 ;$ I 4$)$. Dans $30 \%$ des cas, on observe une translocation dite "variante " où le chromosome 8 échange du matériel avec le chromosome $22, t(8 ; 22)$ ou $2, t(2 ; 8)$. Deux particularités ont d'emblée attiré l'attention, l'une étant la constance avec laquelle ces translocations sont retrouvées ( $100 \%$ des cas), et l'autre la constance des points de cassure : sur le bras long du chromosome 8 (8q24) d'une part, sur le bras long du chromosome i4 (I4932) ou du chromosome 22 (22qII), ou sur le bras court du chromosome 2 (2p I3) d'autre part. Ces remaniements chromosomiques sont spécifiques du lymphome de Burkitt et doivent être distingués des réarrangements aléatoires et non spécifiques habituellement observés dans les lignées cancéreuses.

Le plasmocytome murin chimio-induit est un autre exemple de prolifération monoclonale des lymphocytes $B$ avec translocation spécifique $t(12 ; 15)$ et plus rarement $t(6 ; 15)[8]$. Il constitue un modèle animal équivalent du lymphome de Burkitt humain.

\section{Des chromosomes aux gènes}

L'implication des gènes des immunoglobulines dans le lymphome de Burkitt a été soupçonnée dès que furent caractérisés les trois types de translocations $t(8 ; 14), t(8 ; 22)$ et $t(2 ; 8)$. En effet, entre 1980 et 1982 les gènes des immunoglobulines étaient localisés grâce à l'utilisation de sondes moléculaires récemment clonées : les gènes des chaînes lourdes, mu, delta, gamma, epsilon et alpha, sur le chromosome i4; les gènes de la chaîne légère lambda sur le chromosome 22 et ceux de la chaîne légère kappa sur le chromosome 2. Ainsi localisés sur chacun des chromosomes échangeant du matériel génétique avec le chromosome 8 , les gènes des immunoglobulines apparaissaient comme des partenaires possibles de l'échange. Le modèle du plasmocytome murin était également conforme à ces prévisions : deux translocations étaient, observées $t(12$; I 5$)$ et $t(6 ;$ I 5$)$, les gènes des chaînes lourdes étant, chez la souris, sur le chromosome i2, et ceux de la chaîne kappa sur le chromosome 6 .

Les lignées monoclonales de Burkitt sont constituées par des lymphocytes $B$, seules cellules de l'organisme capables de synthétiser les immunoglobulines. Le typage des immunoglobulines produites par chaque lignée a montré une concordance troublante entre le type de translocation et la nature des chaînes légères produites (un lymphocyte ne peut produire qu'un seul type de chaîne légère, kappa ou lambda, jamais les deux à la fois, ce phénomène est appelé "exclusion isotypique $")$ : dans les translocations $t(8 ; 22)$, les cellules produisaient exclusivement lambda et dans les translocations $t(2 ; 8)$, elles produisaient uniquement kappa; quant aux lymphocytes $t(8 ; 14)$, ils étaient indifféremment lambda ou kappa. Cette concordance, mise en évidence par Lenoir, Preudhomme, Bernheim et Berger dès 198I [9], eut une valeur heuristique considérable car elle indiquait clairement une voie de recherche logique. Une première question simple, celle de la localisation régionale des gènes d'immunoglobulines sur les chromosomes 2, 14 et 22 normaux, fut vite résolue : les gènes kappa étaient localisés en 2pr3, les gènes des chaînes lourdes étaiènt en 14932, ceux de lambda en 22qII, soit très exactement à l'emplacement des points de cassure chromosomique des cellules de Burkitt. Que se passait-il dans ces translocations? Par deux approches différentes, l'hybridation in situ sur des préparations chromosomiques d'une part et l'analyse d'hybrides somatiques homme/rongeur d'autre part, il fut démontré que les gènes d'immunoglobulines étaient partiellement transloqués sur le chromosome 8 [IO-12].

Cette découverte était un premier pas dans la démarche convergente de la cytogénétique et de la biologie moléculaire que nous avons évoquée plus haut : pour la première fois un 


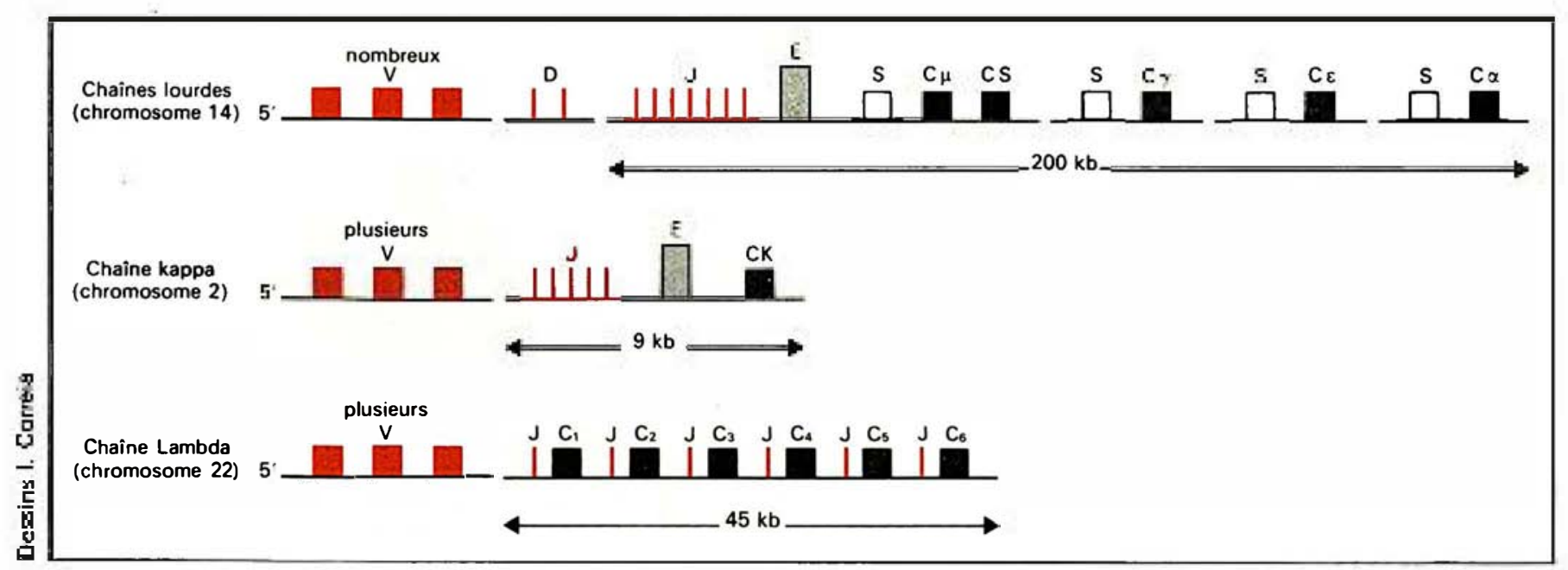

point de cassure chromosomique pouvait être défini à quelques kilobases près. Ultérieurement, grâce au clonage et à l'étude de la séquence de l'ADN des lignées de Burkitt, le niveau de définition a pu atteindre celui du nucléotide.

\section{Recombinatoire des gènes}

La diversité quasi illimitée des anticorps s'explique par une constitution en éléments discontinus et éloignés sur le génome dans l'état dit "germinal" ou quiescent, secondairement juxtaposés par le jeu de recombinaisons somatiques aléatoires se produisant seulement au cours de la maturation des immunocytes $\mathrm{B}\left[\mathrm{I}_{3}\right]$. Le résultat obtenu par cette combinatoire complexe est la formation de gènes fonctionnels pour les chaînes lourdes, la chaîne légère kappa et la chaîne légère lambda des immunoglobulines $(f i g . l)$. Les éléments séparés dans l'état germinal ne sont en principe pas transcrits. Seule la version finie est transcrite en utilisant un promoteur situé juste en amont de la région $\mathrm{V}$ réarrangée. On explique l'activation de ce promoteur par le rapprochement du complexe variable qui le porte et d'une séquence cis-activatrice dite "enhancer". située en amont de la région constante. En définitive, ce n'est que dans sa version finie, réarrangée, qu'un gène d'immunoglobuline est transcrit. L'étude de la structure des gènes d'immunoglobulines dans les trois types de translocations $\mathrm{m} / \mathrm{s}$ mars 85

Figure 1. Schéma simplifié des gènes d'immunoglobulines avant réarrangement.

Les éléments entrant dans la constitution de chaque gène d'immunoglobuline sont une région variable $(V)$ et une région constante $(C)$. La région variable est elle-même morcelée en éléments $V$ (plusieurs centaines de copies différentes pour les chaînes lourdes), éléments $D$ (plusieurs dizaines, pour les chaînes lourdes seulement) et éléments 7 (cinq ou six copies). Le schéma représente l'état basal où tous ces éléments sont éloignés les uns des autres.

Des recombinaisons aléatoires aboutissent au rapprochement $V D$ et 7 (ou $V$ et f) pour former un domaine variable actif (non représenté ici). Celui-ci se juxtapose ensuite, immédiatement en $5^{\prime} d^{\prime}$ un gène $C$ (au niveau d'une région "switch " $=S$ dans le cas des chaines lourdes), et l'ensemble est transcrit en un messager unique.

Il est probable que ces mouvements de gènes, qui sont des résultats de recombinaisons au niveau d'un même chromosome, favorisent des recombinaisons illégitimes, notamment avec le chromosome 8 . E. = "enhancer".

ubservés dans le lymphome de Burkitt a révélé les informations suivantes : dans la translocation $t(8 ; \mathrm{I} 4)$, ce sont les gènes $\mathrm{V}$ des chaînes lourdes qui sont totalement ou partiellement transloqués sur le chromosome $8[\mathrm{r} 0]$ et dans les translocations variantes $t(8 ; 22)$ et $t(2 ; 8)$, ce sont les gènes $C$ des chaînes légères qui sont transposés totalement ou partiellement sur le chromosome 8 [i I, I2]. Dans presque tous les cas, les régions $\mathrm{C}$ et $\mathrm{V}$ se retrouvent sur deux chromosomes différents. Il reste à savoir s'il existe un gène particulier du chromosome 8 qui participerait à l'échange.

\section{A la recherche}

Dans le courant de l'année i982, les premières localisations chromosomiques des oncogènes ont été publiées. Dès que la localisation de l'oncogène $c$-myc sur le chromosome 8 fut connue, sa participation aux translocations du lymphome de Burkitt fut activement recherchée. La localisation précisément en 8q24 du gène $c$-myc en faisait un candidat sérieux. De plus, on venait de découvrir le mécanisme du lymphome $B$ aviaire induit par le. virus $A L V$ : il s'agit de l'activation du proto-oncogène cellulaire $c$-myc par insertion d'un promoteur viral à proximité[14]. Dans les lignées de Burkitt avec translocation $t(8 ; 14)$, le groupe de Leder puis d'autres devaient découvrir des réarrangements de $c$-myc dans deux tiers des cas [ I 5-I 7], ce qui suggérait la participation de ce gène dans l'échange chromosomique. Après clonage moléculaire de l'ADN des différentes lignées, il devenait possible d'identifier les clones contenant à la fois des fragments de gène d'immunoglobuline et de $c$-myc, et d'en déterminer la séquence. 
Le gène $c$-myc normal est constitué par trois exons séparés par deux introns ( fig. 2,3 et 4 ). Seuls les exons 2 et 3 coderaient pour une protéine de 439 amino-acides. L'exon I serait donc non codant, ce qui n'est pas admis par tous [18], et correspondrait à une séquence leader non traduite particulièrement longue de l'ARN messager. Cet exon est absent du gène $v$-myc du rétrovirus aviaire $M C-29$ responsable de sarcomes aviaires à évolution rapide. On en déduit que l'exon I pourrait exercer une fonction de régulation sur l'expression du gène $c$-myc et que son amputation conduirait à une transcription constitutive, donc à une activation incontrôlée. Dans les lymphomes de Burkitt avec $t(8 ;$ I4), $c$-myc est transloqué sur le chromosome 14 où il est fusionné avec un gène de la région constante des chaînes lourdes d'immunoglobulines (fig. 2). Le point de jonction est d'une remarquable diversité d'une lignée à l'autre, ce qui signifie que le siège de la cassure chromosomique peut varier. Quelles sont les conséquences fonctionnelles des réarrangements d'immunoglobulines et des réarrangements de $c$-myc occasionnés par la translocation?

Grâce aux hybrides somatiques homme/rongeur, le groupe de Croce a pu obtenir une réponse claire dans les lignées $t(8 ; 14)$ : le gène $c$-myc juxtaposé à un gène d'immunoglobuline s'exprime de manière constitutive, alors que le gène $c$-myc du chromosome 8 normal n'est pas transcrit [19]. Ceci indique que dans sa nouvelle position le gène $c$-myc

\section{RÉFÉRENCES}

18. Gazin C., Dupont de Dinechin S., Hampe A. et al.. Nucleotide sequence of the human $c$-myc locus: provocative open reading frame within the first exon. Embo 7 1984; 3:383-7.

19. Nishikura K., ar-Rushdi A., Erikson J., Watt R., Rovera G., Croce C. M. Differential expression of the normal and of the translocated human c-myc oncogenes in B cells. Proc Natl Acad Sci $U S A$ 1983; 80 : 4822-6.

20. Davis M., Malcolm S., Rabbitts Th. Chromosome translocation can occur on either side of the $c$-myc oncogene in Burkitt lymphoma cells. Nature 1984; $308: 286-8$.

21. Hollis G. F., Mitchell K. F., Battey J. et al. A variant translocation places the lambda immunoglobulin genes 3 ' to the $c$-myc oncogene in itt's lymphoma. Nature 1 984; 307: 752-5. transloqué est activé en permanence. Il s'agit là du premier effet de position jamais démontré pour un gène humain. Dans les translocations variantes $t(8 ; 22)$ et $t(2 ; 8)$ le gène $c$-myc n'est pas transloqué. Il demeure sur le chromosome 8 et reçoit, plus ou moins loin du côté $3^{\prime}$, des gènes $C$ lambda ou $C$ kappa, provenant respectivement du chromosome 22 ou du chromosome $2[20,2 \mathrm{I}]$. En définitive, que le gène $c$-myc soit transposé sur le chromosome I4 ou qu'il demeure sur le chromosome 8 , il est toujours situé en $5^{\prime}$ d'un gène $C$ d'immunoglobuline (fig. 4).

\section{Activation ou dérepression?}

Malgré la relative diversité des sites de juxtaposition entre $c$-myc et immunoglobulines, la conséquence est univoque : $c$-myc est devenu constitutif. Son expression en ARN varie d'une lignée à l'autre, mais elle est en général significativement

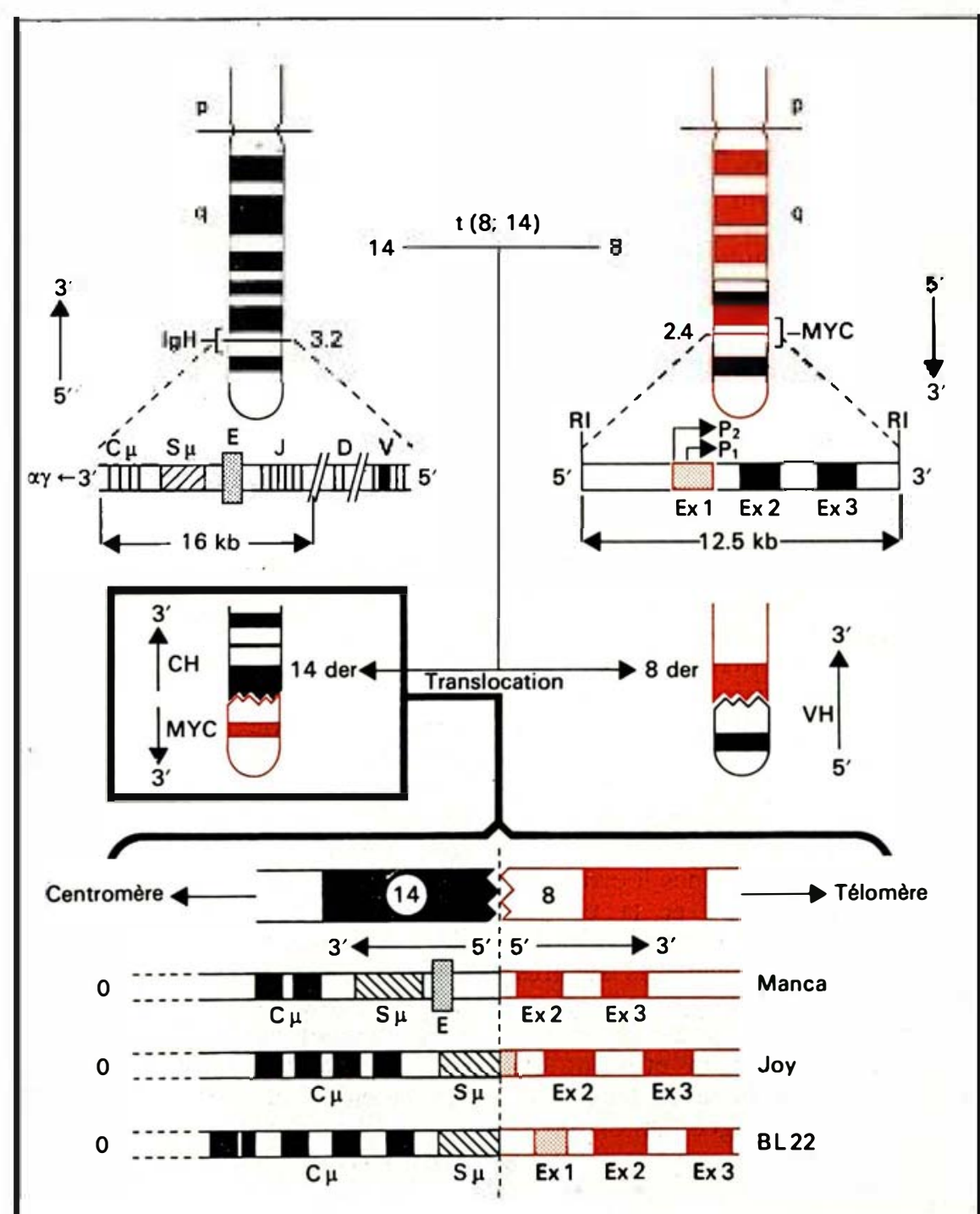

Figure 2. Pathologie moléculaire de la translocation $8 ; 14$.

La translocation transporte le gène c-myc en $5^{\prime}$ d'un gène d'immunoglobuline (Cmu). En haut et à gauche : organisation du chromosome 14 intact et des gènes d'immunoglobulines non réarrangés $(\mathrm{C} m \mathrm{mu})$.

En haut et à droite : organisation du chromosome 8 intact et du gène c-myc normal. Encadré : juxtaposition de c-myc et de la région $C$ mu après translocation $8 ; 14$.

En bas : illustration de la fusion entre c-myc et $C$ mu. Noter l'orientation opposée des 2 gènes et la variété des points de cassure par rapport au $l^{\text {er }}$ exon de c-myc. 


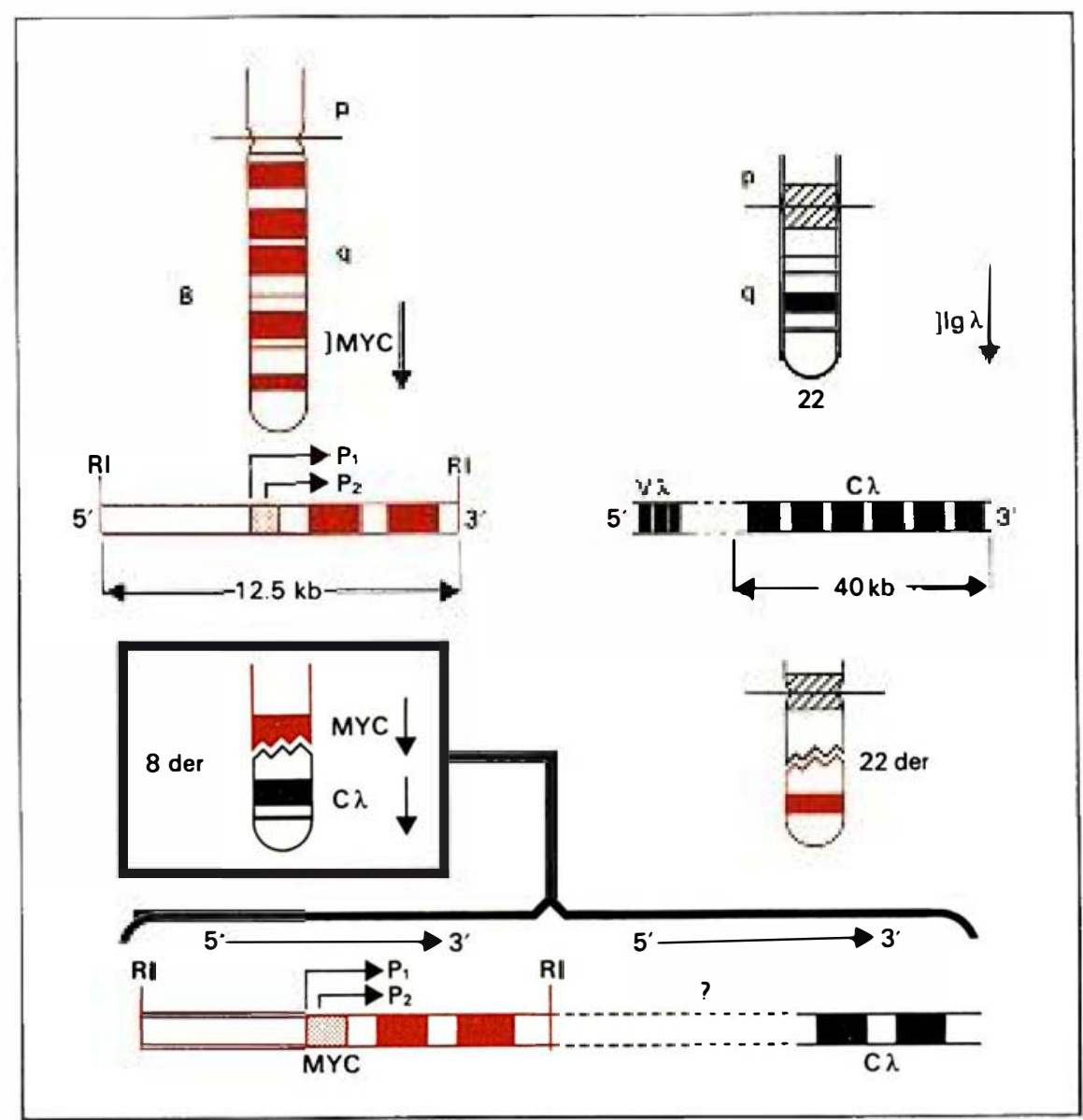

Figure 3. Pathologie moléculaire de la translocation 8; 22. La translocation transporte un gène C lambda en $3^{\prime}$ du gène c-myc. Noter l'orientation identique des deux gènes. ces régulatrices situées dans le gène ou à proximité. La thèse d'une activation permanente, par un effet de position, de certains gènes pouvant jouer un rôle dans le déterminisme du cancer est défendue depuis 1979 par George Klein [22, 23]. Puisque, dans le lymphome de Burkitt, $c$-myc se retrouve toujours placé dans un environnement de gène d'immunoglobuline, on a cherché si une séquence du domaine des immunoglobulines, juxtaposée à $c$-myc, pouvait l'activer. Cette quête est demeurée jusqu'ici infructueuse. D'un autre côté, la théorie d'une dérepression de $c$-myc par échappement à une régulation normale est très séduisante [24]. En effet, l'exon I de $c$-myc est un candidat sérieux à une fonction de régulation. Non exprimée au niveau du produit final, cette séquence a été très conservée au cours de l'évolution. Or, dans un certain nombre de lignées de Burkitt avec $t(8$; I 4$)$, la translocation a décapité totalement ou partiellement l'exon i. Lorsque ce n'est pas le cas, des mutations ponctuelles ont été détectées dans l'exon I et dans son voisinage [2529]. En explorant des lignées avec la translocation variante $t(8 ; 22)$, nous avons nous-mêmes trouvé dans cinq cas sur six de multiples mutations dans l'exon i et dans les séquences adjacentes à cet exon (Szajnert et al., résultats non publiés). Il est tentant de considérer ces mutations somatiques comme capables d'alté-

augmentée. La protéine correspondante a également été trouvée augmentée. Plusieurs hypothèses ont été avancées pour expliquer le dérèglement de $c$-myc dans les cellules de Burkitt. Elles doivent tenir compte de deux faits expérimentaux indiscutables : la juxtaposition du gène $c$-myc et d'un gène constant d'immunoglobuline et l'expression permanente du c-myc juxtaposé, alors que son allèle normal demeure totalement réprimé. Il existe deux courants de pensée, non nécessairement exclusifs d'ailleurs : l'un considère que $c$-myc est devenu constitutif par sa juxtaposition et son asservissement à des gènes d'immunoglobulines, l'autre considère que l'abolition de la régulation de $c$-myc est due à des anomalies structurales intéressant des séquen-

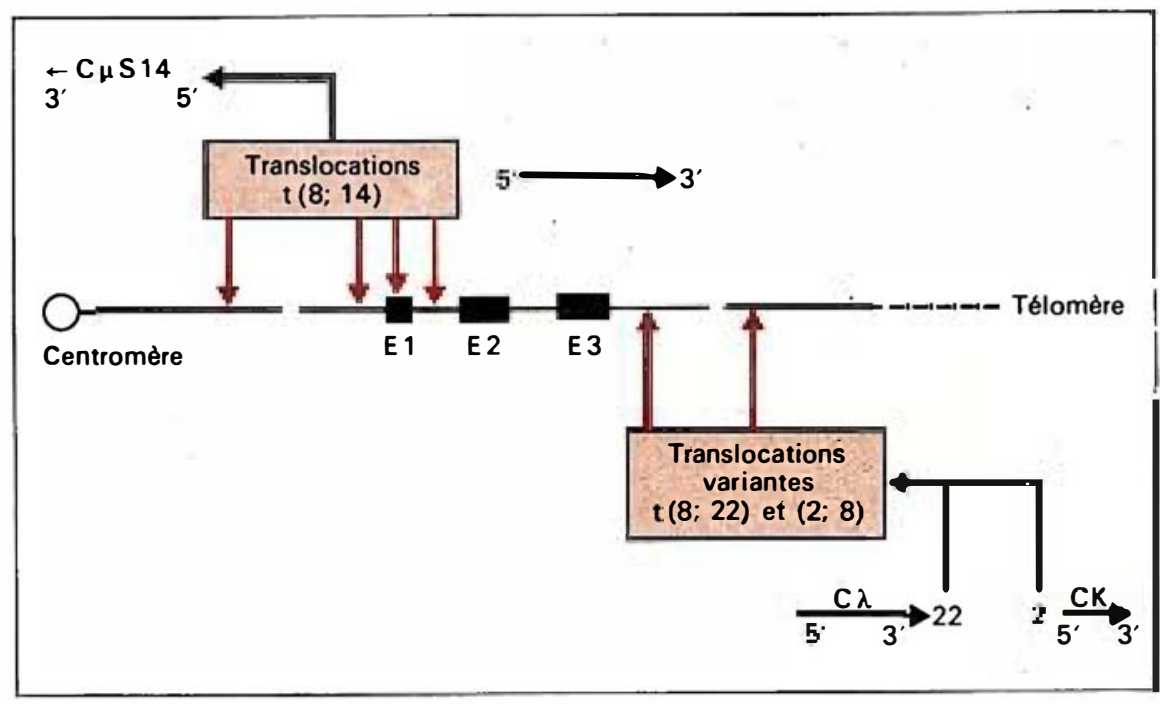

Figure 4. Récapitulation des points de cassure dans les différentes translocations. Dans tous les cas c-myc se retrouve en $5^{\prime}$ d'un gène constant d'immunoglobuline. 


\section{REFEERENCES}

22. Klein G. Lymphoma development in mice and humans: diversity of initiation is followed by convergent cytogenetic evolution. Proc Natl Acad $S c i U S A$ 1979; 76 : 2442-6.

23. Klein $G$. The role of gene dosage and genetic transcription in carcinogenesis. Nature 1981; 294 : 313-8.

24. Leder P., Battey J., Lenoir G. et al. Translocations among antibody genes in human cancer. Science 1983; $222: 765-71$.

25. Rabbitts T. H., Hamlyn P. H., Baer R. Altered nucleotide sequences of a translocated $c$-myc gene in Burkitt lymphoma. Nature 1983; 306 : $760-5$.

26. Rabbitts T. H., Forster A., Hamlyn P., Baer R. Effect of somatic mutation within translocated c-myc genes in Burkitt's lymphoma. Nature 1984; 309: 592-7.

27. Taub R., Moulding C., Battey J. et al. Activation and somatic mutation of the translocated c-myc gene in Burkitt lymphoma cells. Cell 1984 ; $36: 339-48$.

28. Wiman K. G., Clarkson B., Hayday A. C., Saito H., Tonegawa S., Hayward W. S. Activation of a translocated $c$-myc gene: role of structural alterations in the upstream region. Proc Natl Acad Sci USA 1984; 81 : 6798-802.

29. Taub R., Kelly K., Battey J. et al. A novel alteration in the structure of an activated $c-\mathrm{Myc}$ gene in a variant $t(2 ; 8)$ Burkitt lymphoma. Cell 1984; 37 : 5 I 1-20.

30. Toncgawa $S$. Somatic generation of antibody diversity. Nature 1983; 302: 575-81.

31. Croce C. M., Erikson J., ar-Rushdi A., Aden D., Nishikura K. Translocated $c$-myc oncogene of Burkitt lymphoma is transcribed in plasma cells and repressed in lymphoblastoid cells. Proc Natl Acad Sci USA 1984; 81 : 3170-4.

32. Kelly K., Cochran B. A., Stiles C. D., Leder $P$. Cell-specific regulation of the $c$-myc gene by lymphocyte mitogenes and platelet-derived growth factor. Cell $1983 ; 35: 603-10$.

33. Land H., Parada L. F., Weinberg R. A. Cellular oncogenes and multistep carcinogenesis. Science 1983; $222: 771-8$.

34. Diamond A., Cooper G. M., Ritz J., Lane M. $A$. Identification and molecular cloning of the human Blym transforming gene activated in Burkitt's lymphomas. Nature 1983; 305 : 1 1 2-6.

35. Murray M. J., Cunningham J. M., Parada L. F., Dautry F., Lebowitz P., Weinberg R. A. The HL-6o transforming sequence: a ras oncogene coexisting with altered myc genes in hemopoietic tumors. Cell $1983 ; 33: 749-57$.

36. Whittle H. C., Brown J., Marsh K. et al. T-cell control of Epstein-Barr virus-infected B cells is lost during P. falciparum malaria. Nature 1984; 31 2 : 449-50.

\section{TIRÉS A PART}

J. C. Kaplan : Institut de pathologie moléculaire. Inserm (U 129). 24, rue du Faubourg SaintJacques, 75014 Paris. rer la sensibilité à des signaux négatifs, donc d'induire une expression constitutive de $c$-myc.

Comment corréler ces événements somatiques avec la juxtaposition de $c$-myc et d'un gène d'immunoglobuline? Nous avons vu que dans tous les cas $c$-myc est placé en $5^{\prime}$ d'un gène d'une région constante d'immunoglobuline, c'est-à-dire dans un domaine normalement occupé par un gène variable après réarrangement. Or on sait qu'à cet emplacement, les gènes $\mathrm{V}$ subissent des mutations somatiques introduisant un facteur supplémentaire de diversité [30]. Ainsi s'expliquerait une hypermutabilité somatique de $c$-myc, quel que soit le type de translocation.

\section{Une auto-régulation de c-myc?}

Dans le modèle que nous venons de décrire, Leder puis Rabbitts ont suggéré que le produit de $c$-myc lui-même pourrait jouer ce rôle $[24,26]$. Cette auto-régulation expliquerait que la mise en ouvre de $c$-myc soit très limitée dans le temps. En effet, dans les conditions normales, $c$-myc ne s'exprimerait que dans une fenêtre étroite du cycle cellulaire, entre les stades Go et $G_{\text {I. }}$. Une perte de la sensibilité d'un gène $c$-myc à l'inhibition par son produit expliquerait à la fois l'expression constitutive et l'extinction de l'allèle normal présent sur le chromosome 8 non transloqué [24]. En revanche on n'a pas trouvé de mutations dans les parties codantes de $c$-myc (exons 2 et 3 ), ce qui élimine l'hypothèse d'une activation qualitative de $c$-myc à la manière des gènes de la famille Ras.

En définitive, Leder [29] distingue deux types de lymphomes de Burkitt : ceux où une lésion grossière, amputation ou remaniement, suffit pour dérégler $c$-myc [cas des translocations $t(8$; I4) les plus fréquentes] et ceux, beaucoup plus rares, où des réarrangements parf ois lointains induisent une accumulation lente de mutations ponctuelles et finissent à la longue par dérégler $c$-myc [cas des translocations variantes $t(8 ; 22)$ et $t(2 ; 8)]$.

Dans une série d'expériences de fusion de cellules de Burkitt avec des cellules murines, plasmocytes, lymphoblastes et fibroblastes, Croce et son équipe ont montré que l'expression apparemment constitutive de $c$-myc était en fait sensible à l'environnement cellulaire $[19,3 \mathrm{I}]$. Cette expression persiste après fusion avec des plasmocytes, mais elle est réprimée après fusion avec des lymphoblastes et des fibroblastes. Ainsi persisterait au niveau de $c$-myc une possibilité de régulation par des facteurs tissulaires spécifiques agissant en trans. Ces facteurs restent pour l'instant purement hypothétiques.

Il faut reconnaître qu'à l'heure où ces lignes sont écrites, il n'existe aucun modèle général expliquant de manière satisf ${ }^{f}$ isante comment des anomalies si variées de l'ADN au voisinage du gène $c-m y c$, peuvent engendrer sa dérégulation. La tendance actuelle est de considérer que le produit de $c$-myc agit directement sur l'ADN en intervenant, dans le mécanisme délicat c'e la prolifération cellulaire, Je gène $c$-myc étant lui-même normusiement placé sous la commande, très étroitement temporisée, de facteurs de croissance, dont certains sont produits par des oncogènes ( $c$-sis par exemple) [32]. Depuis les travaux du groupe de Weinberg [33], le gène $c$-myc est considéré comme un ǵène immortalisant, mais insuffisant à lui seul pour conférer à la cellule un phénotype tumoral. Ce dernier ne serait acquis que grâce à la collaboration d'un autre oncogène, appartenant à la famille Ras. Ce schéma s'accorde bien avec la théorie, généralement admise, qui fait du cancer la résultante de plusieurs événements. Qu'en est-il dans le lymphome de Burkitt? L'ADN des cellules de Burkitt possède effectivement une activité transformante sur la lignée fibroblastique murine $3 \mathrm{~T}_{3}$, mais après transfections successives $c$-myc n'est pas retrouvé dans l'ADN transformant. La nature précise de la séquence responsable reste controversée. Pour Cooper [34], il s'agirait d'un équivalent humain du gène $B-l y m_{I}$, découvert initialement dans des lymphomes de poulet. Pour Weinberg, il s'agirait du gène $\mathrm{N}$-ras activé trouvé dans la lignée de Burkitt Ramos et également dans d'autres cancers humains [35]. Bien qu'ouvert depuis 
l'été 1983 , le débat n'a encore reçu aucune pièce nouvelle à verser au dossier, et le problème du second oncogène reste entier.

Les réarrangements géniques présidant à la synthèse des immunoglobulines pourraient accidentellement juxtaposer des gènes d'immunoglobulines (région $\mathrm{C}$ essentiellement) et d'autres gènes à la faveur d'homologies de séquence. Une recombinaison illégitime entre $c$-myc et un gène d'immunoglobuline pourrait donc être l'événement déterminant la translocation. Si cet événement aboutit à une expression dérégulée de $c$-myc, et seulement à cette condition, la cellule où il s'est produit aurait un avantage sélectif et constituerait un clone malin. Comment faire intervenir les facteurs épidémiologiques qui semblent déterminants dans certaines régions du monde et qui avaient attiré l'attention de Burkitt? Un très récent travail de Whittle et al. [36] éclaire ce problème d'un jour nouveau. Ces auteurs ont constaté que les accès palustres occasionnés par le Plasmodium falciparum entraînent une augmentation des cellules $T$ suppresseurs et une baisse des cellules $T$ helper. Il s'ensuit une prolifération non ou mal contrôlée des lymphocytes B, ce qui expliquerait l'hyperproduction d'immunoglobulines observée chez les enfants atteints de lymphome de Burkitt africain. L'hyperplasie du pool des lymphocytes B et la synthèse accrue d'immunoglobulines augmenterait les chances de recombinaison accidentelle entre $c$-myc et gènes d'immunoglobulines. $\mathrm{La}$ figure 5 représente un schéma plausible faisant intervenir le virus EB et le paludisme avant la translocation.

Le lymphome de Burkitt constitue un véritable paradigme où s'entremêlent de très nombreuses questions d'actualité. L'engouement dont il a fait brusquement l'objet de la part des biologistes moléculaires à partir de 1982 n'est pas près de cesser. Si l'un des objectifs initiaux : faire converger cytogénétique et biologie moléculaire a été atteint, ce modèle devrait continuer à mobiliser les efforts des chercheurs désirant comprendre le rôle des oncogènes dans le déterminisme des cancers et dans la cinétique cellulaire, déchiffrer les mécanismes du réarrangement des immunoglobulines et de la régulation des lymphocytes $B$, découvrir le rôle du virus d'Epstein-Barr dans le détermi-

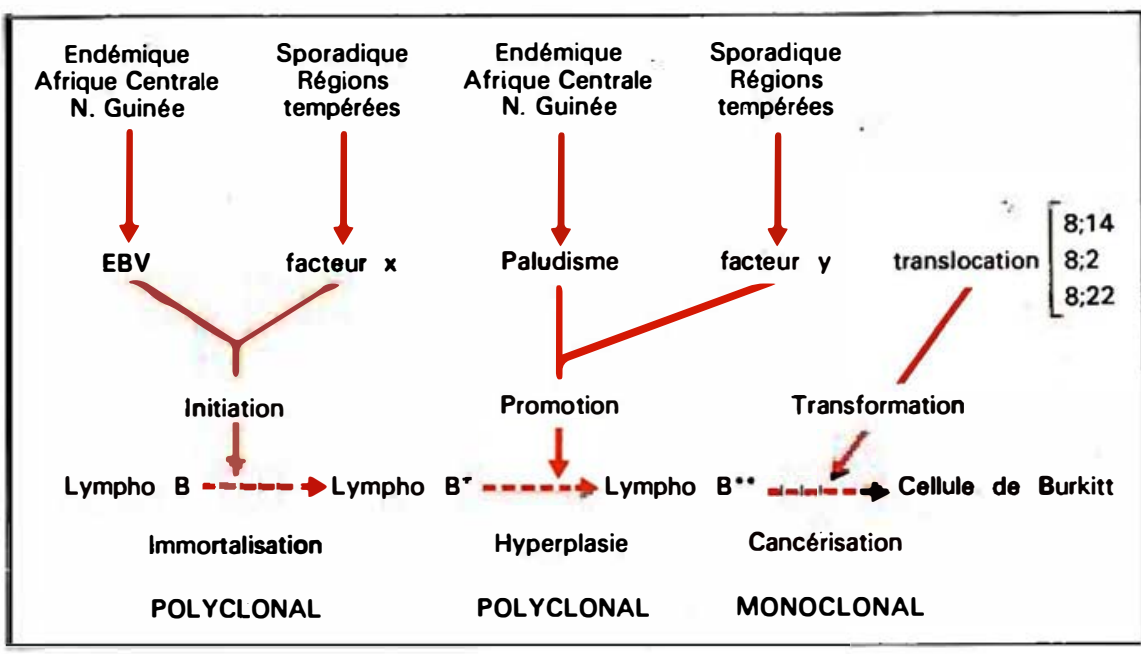

Figure 5. Schéma hypothétique de la séquence des événements susceptibles d'aboutir à la formation d'une cellule de Burkitt.

On admet que l'infection par le virus EB est un premier événement, polyclonal, qui immortaliserait un certain nombre de lymphocytes $B$. Un second événement, lié au paludisme, est la prolifération, polyclonale, de ces lymphocytes qui échapperaient au contrôle par les lymphocytes $T$. Un troisième événement, monoclonal, serait la translocation provoquée par une recombinaison accidentelle entre c-myc et des gènes $C$ d'immunoglobulines. Notons que pour les lymphomes de Burkitt sporadiques les rôles des virus $E B$ et du paludisme sont tenus par d'hypothétiques facteurs $X$ et $Y$. nisme du lymphome, premier cancer humain apparemment viroinduit

\section{Summary}

Burkitt's Lymphoma (BL) is a monoclonal malignant proliferation of human B lymphocytes (immunoglobulin making cells). In tropical countries, like Uganda where it was first described by Burkitt, the disease is endemic and in most cases associated with EpsteinBarr virus (EBV). In other countries the disease is sporadic and rarely associated with $\mathrm{EBV}$. In all cases, irrespective of the geographic distribution and EBV association, there is a balanced translocation between chromosome 8 and a chromosome carrying immunoglobulin (Ig) genes, i. e. chromosome 14 (heavy chains genes), chromosome 2 (light chain kappa genes) and chromosome 22 (light chain lambda genes). By using specific genes probes it was found that in the common $\mathrm{BL}$ with the $8: 14$ translocation $(70 \%$ of the cases) the variable subset of heavy chain Ig genes is transposed onto chromosome 8 whereas the cellular oncogene $c$-myc is transposed onto the chromosome I4 next to a constant heavy chain Ig gene. In all cases $c$-myc is positioned at the $5^{\prime}$ flanking side of a constant Ig gene, and this abnormal juxtaposition is responsible for a constitutive (permanent) activation of $c$-myc. It is believed that this deregulation is promoted by DNA abnormalities resulting from the translocation. The BL represents an exceptional model in which, for the first time in a higher organism, a position effect has been found. It has helped to fill the gap between cytogenetics and molecular biology, because after molecular cloning of BL DNA it has been possible to define the fusion site at the nucleotide level. 